\title{
HOMOTOPY, DIFFEOMORPHISM, AND DEFORMATION CLASSIFICATIONS OF CERTAIN SURFACES OF CLASS VII
}

\author{
ZHENBO QIN \\ (Communicated by Clifford J. Earle, Jr.)
}

\begin{abstract}
We study the homotopy, diffeomorphism, and deformation classifications of all known surfaces of class VII which are not Hopf surfaces.
\end{abstract}

\section{INTRODUCTION}

Let $S_{1}$ and $S_{2}$ be two surfaces (all surfaces in this paper will be smooth compact complex surfaces). They are defined to be deformation equivalent if there exist connected complex spaces $\mathscr{X}$ and $T$ and a smooth proper holomorphic map $\Phi: \mathscr{X} \rightarrow T$, together with points $t_{1}, t_{2} \in T$, such that $\Phi^{-1}\left(t_{i}\right) \cong S_{i}$. It is well known that if $S_{1}$ and $S_{2}$ are deformation equivalent, then they are diffeomorphic.

Let $\mathscr{E}^{\prime}$ be the set of surfaces with nonnegative Kodaira dimensions and blowups of Hopf surfaces. One of the main results in [2] is that given any smooth and oriented 4-manifold $M^{4}$, there exist at most finitely many deformation equivalence classes of surfaces in $\mathscr{E}^{\prime}$ which are diffeomorphic to $M^{4}$.

In this paper, we study the homotopy, diffeomorphism, and deformation classifications of those surfaces not included in $\mathscr{E}^{\prime}$, that is, blow-ups of surfaces of class VII which are not Hopf surfaces. Due to the lack of a complete classification for surfaces of class VII, we concentrate on the set $\mathscr{E}$ of blow-ups of all known surfaces of class VII which are not Hopf surfaces. Our main result (Corollary 3.6) says that given any smooth and oriented 4-manifold $M^{4}$, there exist at most finitely many deformation equivalence classes of surfaces in $\mathscr{E}$ homotopic to $M^{4}$.

\section{Classifications of InOUe SURfaces With $b_{2}=0$}

2.1. Inoue surfaces with $b_{2}=0$ (see [3]).

(a) Surfaces $S_{M}^{+}$and $S_{M}^{-}$. Let $M=\left(m_{i, j}\right) \in \operatorname{SL}(3, \mathbf{Z})$ with eigenvalues $\alpha, \beta$, and $\bar{\beta}$ such that $\alpha>1$ and $\beta \neq \bar{\beta}$. Choose a real eigenvector $\left(a_{1}, a_{2}, a_{3}\right)$

Received by the editors August 23, 1991.

1991 Mathematics Subject Classification. Primary 32J15.

Key words and phrases. Deformation equivalent, surfaces of class VII, Hopf surfaces, Inoue surfaces, surfaces with global spherical shells. 
of $\alpha$ and an eigenvector $\left(b_{1}, b_{2}, b_{3}\right)$ of $\beta$. Let $\mathbf{H}$ be the upper half of the complex plane. Define $G_{M}^{+}$to be the group of analytic automorphisms of $\mathbf{H} \times \mathbf{C}$ generated by

$$
\begin{aligned}
& g_{0}:(w, z) \rightarrow(\alpha w, \beta z), \\
& g_{i}:(w, z) \rightarrow\left(w+a_{i}, z+b_{i}\right) \text { for } i=1,2,3 .
\end{aligned}
$$

Then the quotient $S_{M}^{+}=\mathbf{H} \times \mathbf{C} / G_{M}^{+}$is a surface of class VII.

Similarly, by using $\bar{\beta}$ and $\left(\bar{b}_{1}, \bar{b}_{2}, \bar{b}_{3}\right)$, one obtains $G_{M}^{-}$and $S_{M}^{-}$.

(b) Surfaces $S_{N, p, q, r ; t}^{+}$. Let $N=\left(n_{i, j}\right) \in \mathrm{SL}(2, \mathbf{Z})$ with two real eigenvalues $\alpha$ and $1 / \alpha(\alpha>1)$. Choose real eigenvectors $\left(a_{1}, a_{2}\right)$ and $\left(b_{1}, b_{2}\right)$ of $\alpha$ and $1 / \alpha$, respectively. Fix integers $p, q, r(r \neq 0)$ and a complex number $t$. Define $\left(c_{1}, c_{2}\right)$ to be the solution of

$$
\left(c_{1}, c_{2}\right)=\left(c_{1}, c_{2}\right) \cdot{ }^{t} N+\left(e_{1}, e_{2}\right)+\frac{b_{1} a_{2}-b_{2} a_{1}}{r} \cdot(p, q),
$$

where $e_{i}=n_{i, 1} n_{i, 2} b_{1} a_{2}+\left[n_{i, 1}\left(n_{i, 1}-1\right) a_{1} b_{1}+n_{i, 2}\left(n_{i, 2}-1\right) a_{2} b_{2}\right] / 2(i=1,2)$. Let $G_{N, p, q, r ; t}^{+}$be the group of analytic automorphisms of $\mathbf{H} \times \mathbf{C}$ generated by

$$
\begin{aligned}
& g_{0}^{+}:(w, z) \rightarrow(\alpha w, z+t), \\
& g_{i}^{+}:(w, z) \rightarrow\left(w+a_{i}, z+b_{i} w+c_{i}\right) \text { for } i=1,2, \\
& g_{3}^{+}:(w, z) \rightarrow\left(w, z+\left(b_{1} a_{2}-b_{2} a_{1}\right) / r\right) .
\end{aligned}
$$

Then the quotient $S_{N, p, q, r ; t}^{+}=\mathbf{H} \times \mathbf{C} / G_{N, p, q, r ; t}^{+}$is a surface of class VII.

(c) Surfaces $S_{L, p, q, r}^{-}$. Let $L=\left(l_{i, j}\right) \in \mathrm{GL}(2, \mathbf{Z})$ with $\operatorname{det}(L)=-1$ having two real eigenvalues $\alpha$ and $-1 / \alpha(\alpha>1)$. Choose real eigenvectors $\left(a_{1}, a_{2}\right)$ and $\left(b_{1}, b_{2}\right)$ of $\alpha$ and $-1 / \alpha$, respectively. Fix integers $p, q, r(r \neq 0)$, and define $\left(c_{1}, c_{2}\right)$ to be the solution of

$$
-\left(c_{1}, c_{2}\right)=\left(c_{1}, c_{2}\right) \cdot{ }^{t} L+\left(e_{1}, e_{2}\right)+\frac{b_{1} a_{2}-b_{2} a_{1}}{r} \cdot(p, q),
$$

where $e_{i}=l_{i, 1} l_{i, 2} b_{1} a_{2}+\left[l_{i, 1}\left(l_{i, 1}-1\right) a_{1} b_{1}+l_{i, 2}\left(l_{i, 2}-1\right) a_{2} b_{2}\right] / 2 \quad(i=1,2)$. Let $G_{L, p, q, r}^{-}$be the group of analytic automorphisms of $\mathbf{H} \times \mathbf{C}$ generated by

$$
\begin{aligned}
& g_{0}^{-}:(w, z) \rightarrow(\alpha w,-z), \\
& g_{i}^{-}:(w, z) \rightarrow\left(w+a_{i}, z+b_{i} w+c_{i}\right) \text { for } i=1,2, \\
& g_{3}^{-}:(w, z) \rightarrow\left(w, z+\left(b_{1} a_{2}-b_{2} a_{1}\right) / r\right) .
\end{aligned}
$$

Then the quotient $S_{L, p, q, r}^{-}=\mathbf{H} \times \mathbf{C} / G_{L, p, q, r}^{-}$is a surface of class VII.

\subsection{Classifications of Inoue surfaces with $b_{2}=0$.}

Lemma 2.1. Let $M, N, L$ be as in (a), (b), and (c), of $\S 2.1$, respectively. Then

(i) $S_{M}^{+}$and $S_{M}^{-}$are diffeomorphic, but not deformation equivalent;

(ii) $G_{M}^{+}, G_{N, p, q, r ; t}^{+}$, and $G_{L, p^{\prime}, q^{\prime}, r^{\prime}}^{-}$are pairwisely nonisomorphic.

Proof. (i) is proved by Inoue (see [7]).

(ii) From the definitions of $G_{M}^{+}, G_{N, p, q, r ; t}^{+}$, and $G_{L, p^{\prime}, q^{\prime}, r^{\prime}}^{-}$, we have

(a) $g_{0} g_{i} g_{0}^{-1}=g_{1}^{m_{i, 1}} g_{2}^{m_{i, 2}} g_{3}^{m_{i, 3}}$ and $g_{i} g_{j}=g_{j} g_{i}$ for $i, j=1,2,3$; 
(b) $g_{3}^{+}$commutes with $g_{i}^{+}$for $i=0,1,2$,

$g_{0}^{+} g_{1}^{+}\left(g_{0}^{+}\right)^{-1}=\left(g_{1}^{+}\right)^{n_{i, 1}}\left(g_{2}^{+}\right)^{n_{1,2}}\left(g_{3}^{+}\right)^{p}$,

$g_{0}^{+} g_{2}^{+}\left(g_{0}^{+}\right)^{-1}=\left(g_{1}^{+}\right)^{n_{2,1}}\left(g_{2}^{+}\right)^{n_{2,2}}\left(g_{3}^{+}\right)^{q}$,

$\left(g_{1}^{+}\right)^{-1}\left(g_{2}^{+}\right)^{-1} g_{1}^{+} g_{2}^{+}=\left(g_{3}^{+}\right)^{r}$;

(c) $g_{3}^{+}$commutes with $g_{i}^{-}$for $i=0,1,2$,

$g_{0}^{-} g_{1}^{-}\left(g_{0}^{-}\right)^{-1}=\left(g_{1}^{-}\right)^{l_{1,1}}\left(g_{2}^{-}\right)^{l_{1,2}}\left(g_{3}^{-}\right)^{p^{\prime}}$,

$g_{0}^{-} g_{2}^{-}\left(g_{0}^{-}\right)^{-1}=\left(g_{1}^{-}\right)^{l_{2,1}}\left(g_{2}^{-}\right)^{l_{2,2}}\left(g_{3}^{-}\right)^{q^{\prime}}$,

$\left(g_{1}^{-}\right)^{-1}\left(g_{2}^{-}\right)^{-1} g_{1}^{-} g_{2}^{-}=\left(g_{3}^{-}\right)^{r^{\prime}}$.

For any group $G$, let $\lambda(G)=\{g \in G \mid g \bmod [G, G]$ is of finite order $\}$, and let $\mu(G)=G /[G, G]$. Then we obtain the following conclusions:

(d) $\lambda\left(G_{N, p, q, r ; t}^{+}\right)=\left\langle g_{1}, g_{2}, g_{3}\right\rangle \cong \mathbf{Z}^{3}$,

$\lambda\left(G_{N, p, q, r ; t}^{+}\right)=\left\langle g_{1}^{+}, g_{2}^{+}, g_{3}^{+}\right\rangle$,

$\lambda\left(G_{L, p^{\prime}, q^{\prime}, r^{\prime}}^{-}\right)=\left\langle g_{1}^{-}, g_{2}^{-}, g_{3}^{-}\right\rangle$;

(e) $\mu \lambda\left(G_{N, p, q, r ; t}^{+}\right)=\left\langle g_{1}, g_{2}, g_{3}\right\rangle \cong \mathbf{Z}^{3}$,

$\mu \lambda\left(G_{N, p, q, r ; t}^{+}\right) /$Torsion $=\left\langle g_{1}^{+}, g_{2}^{+}\right\rangle \cong \mathbf{Z}^{2}$,

$\mu \lambda\left(G_{L, p^{\prime}, q^{\prime}, r^{\prime}}^{-}\right) /$Torsion $=\left\langle g_{1}^{-}, g_{2}^{-}\right\rangle \cong \mathbf{Z}^{2}$.

By (e), $G_{M}^{+}$cannot be isomorphic to either $G_{N, p, q, r ; t}^{+}$or $G_{L, p^{\prime}, q^{\prime}, r^{\prime}}^{-}$.

Next we show that $G_{N, p, q, r ; t}^{+}$and $G_{L, p^{\prime}, q^{\prime}, r^{\prime}}^{-}$cannot be isomorphic. Assume the contrary, and let $\phi: G_{N, p, q, r ; t}^{+} \rightarrow G_{L, p^{\prime}, q^{\prime}, r^{\prime}}^{-}$be an isomorphism. By conclusions (a)-(e), we notice the following:

(f) $G_{N, p, q, r ; t}^{+} / \lambda\left(G_{N, p, q, r ; t}^{+}\right)=\left\langle g_{0}^{+}\right\rangle \cong \mathbf{Z}$,

$G_{L, p^{\prime}, q^{\prime}, r^{\prime}}^{-} / \lambda\left(G_{L, p^{\prime}, q^{\prime}, r^{\prime}}^{-}\right)=\left\langle g_{0}^{-}\right\rangle \cong \mathbf{Z}$

(g) $\lambda^{2}\left(G_{N, p, q, r ; t}^{+}\right)=\left\langle g_{3}^{+}\right\rangle \cong \mathbf{Z}$

$\lambda^{2}\left(G_{L, p^{\prime}, q^{\prime}, r^{\prime}}^{-}\right)=\left\langle g_{3}^{-}\right\rangle \cong \mathbf{Z}$.

By (f), (c), and (g), there exist integers $a_{0}= \pm 1, a_{1}, a_{2}$ such that

$$
\phi\left(g_{0}^{+}\right)=\left(g_{0}^{-}\right)^{a_{0}}\left(g_{1}^{-}\right)^{a_{1}}\left(g_{2}^{-}\right)^{a_{2}} \bmod \lambda^{2}\left(G_{L, p^{\prime}, q^{\prime}, r^{\prime}}^{-}\right) .
$$

By (e), there exists $A=\left(a_{i, j}\right) \in \mathrm{GL}(2, \mathbf{Z})$ with $\operatorname{det}(A)= \pm 1$ such that

$$
\phi\left(g_{i}^{+}\right)=\left(g_{1}^{-}\right)^{a_{i, 1}}\left(g_{2}^{-}\right)^{a_{i, 2}} \bmod \lambda^{2}\left(G_{L, p^{\prime}, q^{\prime}, r^{\prime}}^{-}\right) \text {for } i=1,2 .
$$

Applying $\phi$ to the second and third identities in (b) and using the second and third identities in (c), we conclude that $N \cdot A=A \cdot L^{a_{0}}$. Thus,

$$
1=\operatorname{det}(N)=\operatorname{det}\left(L^{a_{0}}\right)=\operatorname{det}(L)=-1,
$$

a contradiction. Therefore $G_{N, p, q, r ; t}^{+}$and $G_{L, p^{\prime}, q^{\prime}, r^{\prime}}^{-}$are not isomorphic.

Lemma 2.2. Let $S$ be an Inoue surface with $b_{2}=0$.

(i) If $\pi_{1}(S)$ is isomorphic to $G_{M}^{+}$, then $S$ is either $S_{M}^{+}$or $S_{M}^{-}$.

(ii) If $\pi_{1}(S)$ is isomorphic to $G_{N, p, q, r ; t}^{+}$, then $S$ is $S_{N, p, q, r ; t}^{+}$.

(iii) If $\pi_{1}(S)$ is isomorphic to $G_{L, p, q, r}^{-}$, then $S$ is $S_{L, p, q, r}^{-}$.

Proof. (i) This is also proved by Inoue (see [7]).

(ii) By Lemma 2.1(ii), the Inoue surface $S$ must be of the form $S_{N^{\prime}, p^{\prime}, q^{\prime}, r^{\prime} ; t^{\prime}}^{+}$ From the arguments in $\S 8$ of [3], we see that the ordered pairs $\left(N^{\prime}, p^{\prime}, q^{\prime}, r^{\prime}, t^{\prime}\right)$ and $(N, p, q, r, t)$ must be equal. Thus, $S$ is $S_{N, p, q, r ; t}^{+}$. 
(iii) This follows from Lemma 2.1(ii) and the arguments in $\S 9$ of [3].

Proposition 2.3. Let $S_{1}$ and $S_{2}$ be two Inoue surfaces with $b_{2}=0$. Then

(i) $S_{1}$ and $S_{2}$ are homotopic if and only if they are diffeomorphic;

(ii) $S_{1}$ and $S_{2}$ are deformation equivalent if and only if they are the same.

Proof. (i) Assume that $S_{1}$ and $S_{2}$ are homotopic. Then $\pi_{1}\left(S_{1}\right) \cong \pi_{1}\left(S_{2}\right)$. If $\pi_{1}\left(S_{i}\right)$ is isomorphic to $G_{N, p, q, r ; t}^{+}$, then both $S_{1}$ and $S_{2}$ are $S_{N, p, q, r ; t}^{+}$by Lemma 2.2(ii). If $\pi_{1}\left(S_{i}\right)$ is isomorphic to $G_{L, p^{\prime}, q^{\prime}, r^{\prime}}^{-}$, then both $S_{1}$ and $S_{2}$ are $S_{L, p^{\prime}, q^{\prime}, r^{\prime}}^{-}$by Lemma 2.2(iii). If $\pi_{1}\left(S_{i}\right)$ is isomorphic to $G_{M}^{+}$, then $S_{i}$ $(i=1,2)$ is either $S_{M}^{+}$or $S_{M}^{-}$by Lemma 2.2(i). From Lemma 2.1(i), we see that $S_{M}^{+}$and $S_{M}^{-}$are diffeomorphic; therefore, $S_{1}$ and $S_{2}$ are diffeomorphic.

(ii) Assume $S_{1}$ and $S_{2}$ are deformation equivalent. Again, $\pi_{1}\left(S_{1}\right) \cong \pi_{1}\left(S_{2}\right)$. If $\pi_{1}\left(S_{i}\right)$ is isomorphic to $G_{N, p, q, r ; t}^{+}$(respectively, $G_{L, p^{\prime}, q^{\prime}, r^{\prime}}^{-}$), then both $S_{1}$ and $S_{2}$ are $S_{N, p, q, r ; t}^{+}$(respectively, $S_{L, p^{\prime}, q^{\prime}, r^{\prime}}^{-}$). If $\pi_{1}\left(S_{i}\right)$ is isomorphic to $G_{M}^{+}$, then $S_{i}(i=1,2)$ is either $S_{M}^{+}$or $S_{M}^{-}$. By Lemma 2.1(i), $S_{M}^{+}$and $S_{M}^{-}$ are not deformation equivalent; therefore, both $S_{1}$ and $S_{2}$ are either $S_{M}^{+}$or $S_{M}^{-}$.

Theorem 2.4. Let $\mathscr{E}_{1}$ be the set of blow-ups of Inoue surfaces with $b_{2}=0$. Then for a smooth and oriented 4-manifold $M^{4}$, there exist at most two surfaces in $\mathscr{E}_{1}$ which are homotopic to $M^{4}$.

Proof. Assume that $\widetilde{S}$ is the $k$-fold blow-up of an Inoue surface $S$ with $b_{2}=0$ and that $\widetilde{S}$ is homotopic to $M^{4}$. Then $\pi_{1}(S) \cong \pi_{1}(\widetilde{S}) \cong \pi_{1}\left(M^{4}\right)$. By Lemma 2.2 , there exist at most two choices for $S$. Since $\chi\left(\mathscr{O}_{S}\right)=\chi\left(\mathscr{O}_{\widetilde{S}}\right)=0$, by the Riemann-Roch formula, $k=\chi(\widetilde{S})=b_{2}(\widetilde{S})=b_{2}\left(M^{4}\right)$, where $\chi(\widetilde{S})$ is the Euler number of $\widetilde{S}$; therefore, there exist at most two choices for $\widetilde{S}$ (note that there exist exactly two choices for $\widetilde{S}$ if and only if $\pi_{1}\left(M^{4}\right)$ is isomorphic to some $\left.G_{M}^{+}\right)$.

3. Classifications of surfaces of Class VII with GSS AND $b_{2}>0$

Definition 3.1 (see [6]). A subset $\Sigma$ of a compact complex surface $S$ is defined to be a global spherical shell (abbreviated GSS) if

(i) $\Sigma=\left\{\left.(x, y) \in \mathbf{C}^{2}|r<| x\right|^{2}+|y|^{2}<r^{\prime}\right\}$ for two positive numbers $r$ and $r^{\prime}$

(ii) the complement $S-\Sigma$ is connected.

Remark 3.2. The only known minimal surfaces of class VII with $b_{2}>0$ are Inoue surfaces with $b_{2}>0$ (see $\left.[4,5]\right)$ as well as the surfaces $S_{n, \alpha, t}$ where $n \in \mathbf{Z}^{+}, 0<|\alpha|<1$, and $t \in \mathbf{C}^{n}$ (see [4, 6, 1]). All these surfaces contain GSS.

Lemma 3.3 (see [6]). If $S$ contains a GSS, then $S$ is deformation equivalent to the $b_{2}(S)$-fold blow-up of a primary Hopf surface.

Lemma 3.4. Let $S_{1}$ and $S_{2}$ be two surfaces of class VII with GSS. Then the following statements are equivalent.

(i) $S_{1}$ and $S_{2}$ are deformation equivalent. 
(ii) $S_{1}$ and $S_{2}$ are diffeomorphic.

(iii) $S_{1}$ and $S_{2}$ are homotopic.

(iv) $b_{2}\left(S_{1}\right)$ and $b_{2}\left(S_{2}\right)$ are equal.

Proof. We only need to show that (iv) implies (i). Let $b_{2}=b_{2}\left(S_{1}\right)=b_{2}\left(S_{2}\right)$. By Lemma 3.3, $S_{i}(i=1,2)$ is deformation equivalent to the $b_{2}$-fold blow-up of a primary Hopf surface. It is well known that all primary Hopf surfaces are deformation equivalent; therefore, $S_{1}$ and $S_{2}$ are deformation equivalent.

Theorem 3.5. Let $\mathscr{E}_{2}$ be the set of blow-ups of minimal surfaces of class VII with GSS and $b_{2}>0$. Then, for a smooth and oriented 4-manifold $M^{4}$, there exist at most finitely many deformation classes of surfaces in $\mathscr{E}_{2}$ homotopic to $\mathrm{M}^{4}$.

Proof. Assume that $\tilde{S}$ is the $k$-fold blow-up of a minimal surface $S$ of class VII with GSS and $b_{2}(S)>0$ and that $\widetilde{S}$ is homotopic to $M^{4}$. Then

$$
k+b_{2}(S)=k+\chi(S)=\chi(\widetilde{S})=\chi\left(M^{4}\right) ;
$$

thus, there are at most $\chi\left(M^{4}\right)$ choices for $b_{2}(S)$. By Lemma 3.4, there are at most $\chi\left(M^{4}\right)$ deformation equivalence classes for $S$. Since $k \leq \chi\left(M^{4}\right)$, there are finitely many deformation equivalence classes for $\widetilde{S}$.

Corollary 3.6. Let $\mathscr{E}$ be the set of blow-ups of all known minimal surfaces of class VII which are not Hopf surfaces. Then, for a smooth and oriented 4-manifold $M^{4}$, there exist at most finitely many deformation equivalence classes of surfaces in $\mathscr{E}$ which are homotopic to $M^{4}$.

Proof. The only known minimal surfaces of class VII which are not Hopf surfaces are Inoue surfaces (with $b_{2}=0$ and $b_{2}>0$ ) and the surfaces $S_{n, \alpha, t}$ (see [7]). By Remark 3.2, the conclusion follows from Theorems 2.4 and 3.5.

\section{ACKNOWLEDGMENT}

The author is very grateful to Robert Friedman and John Morgan for access to the preliminary version of their book [2].

\section{REFERENCES}

1. I. Enoki, Surfaces of class VII $_{0}$ with curves, Tôhoku Math. J. 33 (1981), 453-492.

2. R. Friedman and J. Morgan, Smooth four-manifolds and complex surfaces (to appear).

3. M. Inoue, On surfaces of class $\mathrm{VII}_{0}$, Invent. Math. 24 (1974), 269-310.

4. _ New surfaces with no meromorphic functions, Proc. Internat. Congr. Math. (Vancouver 1974), vol. 1, 1975, pp. 423-426.

5. __ New surfaces with no meromorphic functions. II, Complex Analysis and Algebraic Geometry, Iwanami Shoten Publ. and Cambridge Univ. Press, 1977, pp. 91-106.

6. M. Kato, Compact complex manifolds containing global spherical shells. I, Proc. Internat. Sympos. on Algebraic Geometry (Kyoto, 1977) (M. Nagato, ed.), Kinokuniya Book-Store, Tokyo, 1978, pp. 45-84. 
7. I. Nakamura, $\mathrm{VII}_{0}$ surfaces and a duality of cusp singularities, Classification of Algebraic and Analytic Manifolds (Katata, 1982), Progr. Math., vol. 39, Birkhäuser, Boston, MA, 1983, pp. 333-378.

Department of Mathematics and Statistics, McMaster University, Hamilton, ONTARIO, CANADA L8S 4K1

Current addresses: Institute for Advanced Study, Princeton, New Jersey 08540

Department of Mathematics, Oklahoma State University, Stillwater, Oklahoma 74078

E-mail address: qinz@mcmaster.ca 